\title{
LA IMPLEMENTACIÓN DEL SISTEMA NACIONAL ANTICORRUPCIÓN EN MÉXICO: BREVE ENSAYO.
}

Miguel Ángel Murillo Aispuro ${ }^{1}$

Sumario: I Introducción.II Desarrollo III. Conclusiones

\section{Resumen}

En este documento se aborda el tema del Sistema Nacional Anticorrupción en México y las dificultades que ha enfrentado en el proceso de su implementación. Si bien, las premisas que le dieron origen, siguen vigentes, es la primera vez que en nuestro país se cuenta con un mecanismo para trabajar en el combate a la corrupción no de manera aislada sino conjuntamente, las autoridades de los tres ordenes de gobierno y la participación activa de la sociedad civil, elemento que en esta lucha es clave y motor para accionar y hacer lo que sea necesario para evitar, que esta estrategia, en el contexto de la alternancia en los Poderes de la Nación, pase a engrosar, la larga lista de los proyectos fallidos al efecto.

Palabras clave: anticorrupción, coordinacion intergubernamental-sociedad civil

\section{Abstract}

This document addresses the issue of the National Anti-Corruption System in Mexico and the difficulties it has faced in the process of its implementation. Although, the premises that gave rise to it, are still in force, it is the first time that in our country there is a mechanism to work in the fight against corruption not in isolation but jointly, the authorities of the three orders of government and the active participation of civil society, an element that in this fight is key and driving force to act and do whatever it takes to prevent, that this strategy, in the context of the alternation in the Powers of the Nation, begins to swell, the Long list of failed projects to that effect.

Key words: anti-corruption, intergovernmental coordination-civil society

\footnotetext{
${ }_{1}^{1}$ Titular de la Secretaría de la Contraloría General del Gobierno del Estado de Sonora,murilloaispuro@gmail.com; miguel.murillo@sonora.gob.mx
} 


\section{INTRODUCCIÓN:}

La corrupción continúa siendo uno de los lastres más difíciles de erradicar en México. Prueba de ello son los cientos de casos exhibidos y documentados por organizaciones civiles, medios de comunicación y entidades de fiscalización superior durante los últimos años.

No solo se trata de un problema en México, también en el resto del mundo. La organización Transparencia Internacional, de acuerdo al IPC 2018 (Índice de Percepción de la Corrupción), "revela la incapacidad de la mayoría de los países para controlar la corrupción, lo que a su vez contribuye a una crisis de la democracia en todo el mundo"' Nuestro país no es la excepción, ubicándose en el lugar 138 de los 180 estudiados a nivel mundial.

Estas evidencias junto con los escándalos de hechos de corrupción reportados por personas y organizaciones civiles, medios de comunicación y otros, agravaron la ya deteriorada confianza de los ciudadanos en sus gobernantes, máxime cuando se percibía una violación a los derechos sociales por parte del mismo Estado o de personajes ligados al Poder.

La apuesta de México por una transformación de fondo en la manera de combatir la corrupción se concentró en el Sistema Nacional Anticorrupción SNA, mediante una serie de reformas estructurales que invocaban la unificación de esfuerzos institucionales para hacerle frente, y con el acierto de que fuera

${ }^{2}$ Véase: Transparency International, El índice de percepción de la corrupción muestra un estancamiento de la lucha contra la corrupción en la mayoría de los países [en linea]disponible en:

https://www.transparency.org/news/pressrelease/el_indice_de_percepcion_de_la_ corrupcion_2018; (consulltado el 27 de febrero de 2019) 
encabezado por ciudadanos reconocidos por sus esfuerzos en su lucha por la corrupción.

Sin embargo, a ya más de cuatro años de su propuesta y dos de su implementación, persisten las demandas ciudadanas que dieron origen al sistema $\mathrm{y}$, en ese mismo tenor, podríamos sugerir que el Estado violenta los derechos sociales de los mexicanos.

\section{DESARROLLO}

\section{A guisa de ejemplo el siguiente caso}

Uriel es un joven de 20 años que quiere estudiar la preparatoria pero no tiene forma de hacerlo. En su momento (2014), el Estado no le dio la oportunidad de ingresar al sistema de bachillerato. Él proviene de una familia de seis integrantes, donde la madre no trabaja y el padre es alcohólico, el hermano mayor de 24 años es padre de un niño de cinco, y su hermana menor de 18 vive en unión libre con un joven de 25 que en apariencia tiene problemas de drogadicción.

El mismo Uriel canalizó sus frustraciones hacia las drogas y hoy tiene síntomas de ansiedad por el desuso. El más pequeño de la familia acaba de ingresar a secundaria, y se las ingenió para hacerse del uniforme reglamentario, porque hubo necesidad de ajustar el que consiguió regalado de un vecino de mayor edad.

Seguramente en México hay muchos casos como el de Uriel y probablemnte el de su hermano menor tambien lo será, en donde la búsqueda de un espacio en el bachillerato forma parte de su plan de vida, como medio para integrarse al sector productivo y contribuir así al bien común. El mismo concepto del bien común abordado por Platón en La República y perfeccionado después por su alumno Aristóteles en La Política, que se convirtiera en objeto de estudio de la Filosofía y base fundamental para otras disciplinas de las Ciencias Sociales modernas. 
La idea de que debe prevalecer el beneficio de la mayoría por sobre los beneficios personales, permea aún hoy en los países en pos de la democracia y, en esa misma intención, las sociedades actuales están intentando privilegiar el respeto a los derechos humanos para alejarse de la discriminación, aunque con serios retrasos y obstáculos perfectamente documentados por la Historia.

En palabras de Hernández Ochoa (2014) el bien común son las "condiciones sociales que permiten y favorecen en los seres humanos llegar al progreso de todos los que formen esa sociedad particular; dinamiza el desenvolvimiento de un orden social justo que armoniza los aspectos individuales y sociales de la vida humana, pero para ello la responsabilidad es de todos definirlo y construirlo". ${ }^{3}$

El bien común ha adquirido no solo importancia para el fortalecimiento de los lazos que unen a las sociedades, sino también se ha constituido como un objetivo a alcanzar en algunos países del mundo a través de la igualdad de oportunidades de los ciudadanos; la estrategia más utilizada en las sociedades modernas.

Esto obedece sin duda a que, entre los integrantes de una sociedad, la igualdad de oportunidades intima con el bien común, porque al ser la igualdad un derecho natural también se involucran las opciones equivalentes para la realización de los planes de vida del ciudadano, entre los cuales están el empleo, la política, la salud, la educación, entre otras.

Ahora bien, es deber del Estado el proporcionar mediante las políticas públicas las condiciones suficientes para garantizar dicha igualdad; cuestión que arroja una primera pregunta: ¿cumple el Estado con ese deber?

3 Véase: Hernández Ochoa,María Doris,La construcción del bien común, responsabilidad de todos,en Milenio Diario, S.A. de C.V.,2014 [en línea] disponible en:

https://www.milenio.com/opinion/maria-doris-hernandez-ochoa/columna-mariadoris-hernandez-ochoa/la-construccion-del-bien-comun-responsabilidad-de-todos; [consultado: 27 de febrero de 2019] 
Nadie está obligado a lo imposible, ni siquiera el Estado; pero hacer lo que esté en sus manos o así lo haya determinado, sí es parte de esa obligatoriedad amén de la estipulada, al menos en papel, por parte del Legislativo al hacer obligatoria -mas no gratuita- la Educación Media Superior-.

En México, así como en muchos otros países del orbe, las políticas públicas se concentran en un documento que engloba los distintos rubros de la responsabilidad del Estado: el Plan Nacional de Desarrollo. Y el principio del federalismo marca que de este texto se deben extraer las estrategias que cada uno de las entidades integrantes de la Federación traducirá en acciones, para alcanzar las metas u objetivos plasmados, por lo que debe existir una aceptable coherencia entre los planes estatales y el nacional. Cada estado contribuye así a la administración de los servicios y recursos destinados al desarrollo y progreso del país. Pero, de aquí surge una nueva pregunta: ¿Cumplen los estados con esta responsabilidad?

La organización del poder público en México permite que en ciertos estados un Ejecutivo local comparta tres años de su gobierno con un presidente y tres años más con uno distinto, lo que implica por lo regular comprensibles desfases en acciones y programas de las políticas públicas al tener tres años en operación: Sonora es uno de estos estados.

En el 2009, Sonora enfrentó la alternancia en el partido político dirigente del Poder Ejecutivo, de tal suerte que los sonorenses eran regidos por un Gobierno federal y estatal del mismo partido político. En ese tenor, el Plan Estatal de Desarrollo 2009-2015 extrajo del Plan Nacional de Desarrollo 2007-2012 las mencionadas estrategias y acciones para el cumplimiento de los objetivos planteados en las políticas públicas nacionales. Una de estas es la Transformación Educativa, que tuvo su equivalente estatal en otra denominada Sonora Educado, y contempla entre sus cinco objetivos uno que es importante para lo que nos ocupa:

${ }^{4}$ Reformas publicadas el 9 de febrero del 2012 en el Diario Oficial de la Federación. 
"reducir las desigualdades regionales, de género y entre grupos sociales en las oportunidades educativas" (PND 2007-2012 (2007): 89).

Este tácito reconocimiento a la búsqueda de la igualdad en oportunidades se orienta hacia el bien común, y en donde, al menos en papel, tanto el Estado mexicano como el estatal cumplen por Ley con establecer políticas públicas con esa orientación; pero ¿qué pasa en la práctica?

Gracias a documentos obtenidos mediante la Ley de Acceso a la Información Pública y facilitados por la organización Estrategia Sonora, dedicada a realizar estudios sobre educación en Sonora, se puede apreciar la dimensión entre lo escrito y lo real.

En el año 2010, el Ejecutivo estatal anunció que a partir del 2011 se haría una "histórica inversión de 3,000 millones de pesos" en obras de infraestructura en dos etapas, para el mejoramiento de los centros escolares de nivel básico. Tal anuncio fue exacerbado al grado de denominar al 2011 como "el año de la transformación".

El Instituto Sonorense de Infraestructura Educativa (ISIE) -la entidad responsable de la ejecución de obras en el sector educativo-, sin mediar evidencias de los criterios para seleccionar los centros educativos que se verían beneficiados por la millonaria inversión, ejerció \$571'340,773.44 pesos en 354 planteles en el año 2011 y $\$ 406$ '480,444.64 pesos en 150 planteles durante el 2012 (Reporte Estrategia Sonora (2014): 13, NP) -Aquí es importante hacer dos apuntes: a) el 2012 fue año de elecciones intermedias en el estado, y b) es interesante la proporción de recursos destinados para un menor número de planteles durante dicho año-.

Según los datos analizados y comprobados por Estrategia Sonora, del total de esos casi mil millones de pesos solo 146 millones fueron invertidos realmente (Reporte Estrategia Sonora (2014): 15, NP). Es decir, solo el $15 \%$ se aplicó en las escuelas. 
El proceso de licitación llevado a cabo por el ISIE implicó la asignación de contratos a pocas empresas, mismas que reportaron de forma periódica sus avances de trabajo con las llamadas "estimaciones de obra", mediando el pago correspondiente por cada avance. Al término del "trabajo realizado", el ISIE lo recibió oficialmente por medio de "actas de entrega de obra", en cuyo formato firman los supervisores de obras y el director general del ISIE, los representantes de la compañía constructora, el director de la escuela y el presidente de la sociedad de padres de familia.

Pero hay evidencias de que los padres de familia y algunos directores se negaron a firmar de recibido porque las obras jamás se realizaron en sus centros escolares (Reporte Estrategia Sonora (2014): 20, NP). En esos casos el ISIE optó por recibirlas directamente a través de sus supervisores, arguyendo que "según la normatividad federal y estatal no es obligatoria la elaboración de una acta de entrega de esta dependencia hacia el plantel educativo" (ISIE. Memorándum No. DO/2113/2014: 18 Jun.)

Esa acción inmoral... pero legal, refleja no solo opacidad en el gasto público del Estado en el sector educativo, sino que además podemos catalogarlo como un fraude del Estado a la Educación y, por ende, un incumplimiento de la política pública estatal y federal, porque con esto no se contribuye a reducir las desigualdades en las oportunidades educativas. Por el contrario, la evidencia del pago de trabajos no realizados infiere, además de una acción delictiva, el beneficio económico para unos cuantos, dando al traste a la intención de buscar la finalidad del bien común ya mencionado.

Los informes de Gobierno presentan cuentas alegres sobre los miles de millones invertidos en la infraestructura del sector educativo, y en diferentes espacios radiofónicos el Ejecutivo estatal sonorense anunció que entre 2014-2015 
se invirtieron tres mil millones de pesos adicionales al programa de Transformación Educativa ${ }^{5}$.

Ante lo que hoy conocemos, nos preguntamos: ¿invertidos en qué?

Si recordamos el apunte sobre la inversión del año 2012 y si como está demostrado no se aplicaron los recursos en los centros educativos pero sí se ejercieron, es decir sí se pagaron, la pregunta obligada sería: ¿para qué se utilizaron entonces?

La coyuntura de que el año 2012 hubiese sido un año electoral no puede pasar desapercibida, máxime si consideramos la interesante proporción de la gran cantidad de recursos destinados a un menor número de escuelas, las pocas empresas ganadoras de las licitaciones y la focalización de recursos en municipios que son cabeceras de un distrito electoral.

Si bien no hay evidencias de que dichos recursos hubiesen sido utilizados para asuntos electorales, la duda se desliza peligrosamente ante la falta de transparencia y rendición de cuentas de la que fue objeto el programa de Transformación Educativa por parte del Instituto Sonorense de Infraestructura Educativa.

Por otro lado, al tratarse supuestamente de recursos estatales los utilizados para el programa en cuestión, se puso en evidencia que se violentó el Reglamento de la Ley de Obras Públicas y Servicios Relacionados con las Mismas para el Estado de Sonora, particularmente todo lo contenido en el Capítulo VII sobre la figura de los Comités de Contraloría Social -como las entidades sociales responsables de verificar que las obras se llevaran a cabo-, puesto que no se

${ }^{5}$ En tres años de la administración 2009-2012, el informe de gobierno habla de una inversión "sin precedente" de 1 mil 819 millones de pesos en la mejora de la infraestructura educativa de Sonora, desde los niveles de educación básica hasta educación superior. (Informe a Sonora 2012; Sonora Educado, pag. 4, Gobierno del Estado de Sonora, 2012). 
integraron dichos comités y además no hay muestras fehacientes de su participación en los procesos de licitación, supervisión y entrega.

Al violar la Ley, el Estado contribuye a: 1) La conformación de un sentimiento de impunidad en la ciudadanía, que ve cada vez más disminuida su confianza en las instituciones por esta clase de corrupción, en donde el Estado, además de ser partícipe, es juez y parte si se quisiera perseguir un delito; y 2) Se abona al hecho de que el Estado contribuye a ampliar las desigualdades de oportunidades de sus gobernados.

Por otra parte, en el plano social el ciudadano observa que mientras las escuelas no presentan mejoras palpables, sí hay beneficios para unos cuantos; élites extractivas, como bien expresan de forma contundente Daron Acemoglu y James Robinson en su libro Por qué fracasan los países. ${ }^{6}$

Creemos que el Estado tiene la responsabilidad de transparentar su gasto público en el sector educativo -lo que no sería mal visto en otros rubros, claro estáy se propone la obligatoriedad permanente de que aquel informe públicamente a la sociedad sobre las inversiones realizadas en dicho sector, particularmente las de obras públicas por parte del Instituto de Infraestructura Educativa del Estado de Sonora ${ }^{7}$.

Este incipiente modelo de datos abiertos conforma una base fundamental para el cumplimiento de la función social del Estado en materia educativa, porque es la sociedad la que debe establecer los parámetros de lo que le es conveniente.

\footnotetext{
${ }^{6}$ Vease Capítulos 4 a 15 Acemoglu Daron, Robinson James A. Porque Fracasan los Paises: Los Origenes del Poder, la Prosperidad y la Pobreza, Ed., Deusto,Barcelona España,2013, pp. 465

${ }^{7}$ Para tal fin,la actual administración estatal implementó el portal Tuobra.mx (Herramienta tecnológica diseñada para la rendición de cuentas y el fácil acceso del ciudadano a la información de las Licitaciones y Obra Pública en el Estado de Sonora. Véase Portal Tu Obra[en línea]disponible en: https://tuobra.sonora.gob.mx
} 
Debe elegir por sí misma qué hacer con los datos que le son presentados, y es de esperarse que pueda motivar cambios sustanciales en los modelos de participación de la ciudadanía en el sector educativo.

Hablamos de una auditoría ciudadana, en donde cualquier persona pueda verificar que las inversiones realmente se están aplicando en las escuelas. Como bien lo dice Schmelkes (1992): “... el ideal consiste en que la comunidad haga propia la escuela, la considere como suya, la apoye y se involucre con ella como agente activo en el proceso permanente de mejoramiento de calidad"8.

El estudio del Banco Mundial sobre las desigualdades en América Latina realizado por Enamorado, Rodríguez-Castelán, Winkler y López-Calva (2014), pone de manifiesto la existencia de una relación estrecha entre desigualdad y criminalidad. A mayor desigualdad mayor criminalidad y viceversa.

Por extensión, podemos inferir entonces que el Estado mismo favorece la criminalidad con acciones como la comentada anteriormente.

Esta preocupante circunstancia nos aleja como Estado y como país no solo del fortalecimiento de las instituciones para crear un modelo nacional de democracia, sino también de la construcción de una ciudadanía que posea sentido de pertenencia. Nos lleva invariablemente también a la conformación de esa cultura del engaño y falto de honestidad que critican férreamente ciudadanos de otros países cuando se refieren a la sociedad mexicana.

Los casos de corrupción por parte del Estado no son poco comunes en México. Durante los últimos años, se han documentado cientos de situaciones similares ocurridas en el país, y no solo en el sector educativo, también en el de salud, seguridad, energía, y varios más.

${ }^{8}$ Schmelkes, S. Hacia una mejor calidad en nuestras escuelas. Biblioteca para la Actualización del Maestro. México, D.F. OEA/SEP,1992,pp97 
En el enfoque de este texto, debe mencionarse a Organizaciones no gubernamentales como Mexicanos Primero, por ejemplo, que dieron a conocer en 2013 un informe relativo al gasto de los estados en materia educativa. Los resultados sorprendieron a todos. En ese reporte, el organismo concluye que la corrupción, el despilfarro y la ineficiencia impiden, condicionan o distorsionan el ejercicio pleno del derecho de aprender de los niños ${ }^{9}$. Además, refiere miles de millones de pesos que han sido utilizados de forma inadecuada en el rubro de Educación.

Recordemos que si bien es el Estado debe garantizar los estándares mínimos de salud, vivienda y educación a todos los ciudadanos como derechos sociales ${ }^{10}$ a ello habría que agregar el derecho a la seguridad- que lamentablemente ha avanzado rápidamente a los primeros lugares en la percepción ciudadana de la problemática nacional respecto a su raquítica prestación.

Sin embargo, las diferencias entre teoría y práctica, entre lo establecido por ley y los hechos, y los publicitados resultados de organizaciones como Transparencia Internacional, que ubicó en el 2018 a México en el lugar 138 de 180 entre los países con el índice de percepción de la corrupción más altos, revelan que nuestro país no ha controlado la corrupción.

${ }_{9}^{9}$ Mexicanos Primero: (Mal)Gasto. Estado de la Educación en México 2013, Ed. Mexicanos Primero Visión 2030, México,2013, pp.4

${ }_{10}$ Acorde a lo mencionado por Pisarello "...al menos desde un punto de vista normativo, el constitucionalismo democrático ha comportado una relectura de Locke en clave igualitaria. En virtud de la misma, se propugnan unos mínimos sociales que, junto a los derechos de libertad, pertenecen a todas las personas, son previos al propio contrato social y, por lo tanto, resultan indisponibles tanto para el Estado como para el mercado..." PISARELLO, Gerardo. Los derechos sociales en el constitucionalismo democrático. Boletín Mexicano de Derecho Comparado, [S.I.], jan. 1998[en línea]. Disponible en:

https://revistas.juridicas.unam.mx/index.php/derecho-comparado/article/view/3546/4242; (consultado el 27 febrero de 2019) 
Y si a lo anterior le agregamos el incremento en la percepción de impunidad, se presentan condiciones sociales propicias para que la ciudadanía valore una violación de los derechos sociales por parte del Estado.

Para soportar lo anterior, se destacan los índices de Control de la Corrupción (CC) y de Percepción de la Corrupción (IPC) en México durante la última década (Tabla 1), en los cuales el país obtiene resultados desfavorables.

Tabla 1. Evolución del CC y del IPC en México, 1996-2017.

\begin{tabular}{|c|c|c|c|c|c|}
\hline & 1996 & 2000 & 2005 & 2010 & 2017 \\
\hline CC (valores entre -2.5 y 2.5 ) & -0.51 & -0.25 & -0.27 & -0.36 & -0.93 \\
\hline IPC (valores entre 0 y 100$)$ & 33 & 33 & 35 & 31 & 29 \\
\hline
\end{tabular}

De igual manera, el porcentaje de actos en que la población fue víctima de la corrupción y presentó denuncia (videTabla 2). Aquí se aprecia que solo una media del $5 \%$ de las personas que fueron víctimas de actos de corrupción denunciaba el hecho y que, combinado con el 45 y $56 \%$ de los mexicanos que consideran inútil o una pérdida de tiempo las denuncias, muestra que la población simplemente ve poca utilidad en denunciar la corrupción.

11 Citada por Comité de Participación Ciudadana en Política Nacional Anticorrupción,Propuesta de la PolíticaNacional Anticorrupción,Diciembre 2018,México,pp.21[en línea]disponible en:

http://sumawebdesarrollo.com/cpcnacional_wp/wpcontent/uploads/2019/04/Propue sta-de-la-Poli\%CC\%81tica-Nacional-Anticorrupcio\%CC\%81n.pdf 
Tabla 2. porcentaje de actos en que la población fue víctima de la corrupción y presentó denuncia (2011-2017)

\begin{tabular}{llll}
\hline & 2011 & 2015 & 2017 \\
& & & \\
& $4.40 \%$ & $6.81 \%$ & $3.76 \%$ \\
Denuncias de corrupción & & \\
\hline $\begin{array}{l}\text { Fuente: INEGI, ENCIG, 2011, 2015, 2017. No se incluyen las respuestas de la ENCIG 2013, ya que } \\
\text { dicha pregunta no fue incluida en el cuestionario. }{ }^{12}\end{array}$
\end{tabular}

\section{Iniciativa de Coordinación}

Ante todo este tipo de situaciones y muchas otras donde se exhibió el abuso del poder por parte de personajes ligados al Estado en sus tres ordenes de gobierno, ante la ausencia de castigo a los responsables y ante la debildad institucional de las entidades creadas previamente para combatir la corrupción, el Estado mexicano apostó por promover una iniciativa de coordinación para hacerle frente a la corrupción, dejándole la dirección a los ciudadanos: el Sistema Nacional Anticorrupción.

La intención de esta nueva estrategia técnicamente nació del llamado Pacto por México. Este acuerdo entre las distintas fuerzas políticas del país firmado en 2012 entre Enrique Peña Nieto y los presidentes de los partidos políticos del país, tuvo como objetivo buscar construir un nuevo modelo de sociedad de derechos y más igualitaria, y se sustentó en las aportaciones de la OCDE en materia de reformas estructurales (Getting It Right).

Dentro de los tres ejes rectores, cinco acuerdos y 95 compromisos, se desprendieron las propuestas para realizar las reformas constitucionales que permitieran a su vez traducirlas en políticas públicas del Estado, destacándose el Acuerdo 4. Para la Transparencia, Rendición de Cuentas y Combate a la Corrupción, que da origen a las reformas del 7 de febrero del 2014 en materia de

${ }^{12}$ Citada por Comité de Participación Ciudadana en Política Nacional Anticorrupción... op.cit.,pp.23 
transparencia y la del 27 de mayo del 2015 en materia de corrupción, y el compromiso 85, que menciona la creación del Sistema Nacional Anticorrupción.

Durante este periodo de acuerdos y reformas que le ganaron a México el reconocimiento internacional ${ }^{13}$, las exhibiciones de supuestos actos de corrupción continuaron. En 2015, por ejemplo, Mexicanos Primero hizo un llamado al Estado para atender y solucionar las irregularidades que había expuesto dos años antes y cuya práctica continuaba, en el siguiente tenor.

Por ello, hacemos un llamado: Al Gobierno de la República para que garantice las condiciones para la adecuada aplicación de la ley en todo el territorio nacional, sin excepción alguna. A la Secretaría de Hacienda y Crédito Público SHCP para que asegure el buen uso del presupuesto. A la Secretaría de Educación Pública SEP para que realice un monitoreo eficaz de la información que le hacen llegar los estados y transparente el uso de cada peso del Fondo para la Nómina Educativa FONE ${ }^{14}$. A los

\footnotetext{
${ }^{13}$ Es interesante consultar la opinión de la OCDE respecto a cómo las reformas estructurales del país entre el 2013-2016 contribuyeron al mejor desempeño económico, comparativamente con otros países.Véase:OCDE,Getting it Right Prioridades estratégicas para México,Editions OCDE París,2018 [enlínea]disponible en: https://read.oecd-ilibrary.org/development/getting-it-right_9789264292871es\#page1 ${ }^{14}$ Apócope del Fondo de Aportaciones para la Nómina Educativa y el Gasto Operativo, con la reforma al Artículo 3 de la Constitución Política de los Estados Unidos Mexicanos del 26 de febrero de 2013, misma que estableció como derecho de los mexicanos el recibir una educación de calidad, comenzó un proceso de reformas a la legislación reglamentaria, incluyendo la promulgación de dos nuevas leyes federales: la Ley del Instituto Nacional para la Evaluación de la Educación (Ley del INEE) y la Ley del Servicio Profesional Docente. Este proceso de reformas también afectó a la Ley de Coordinación Fiscal, pues conllevó a la sustitución del Fondo de Aportaciones para la Educación Básica y Normal por un nuevo fondo, el de Aportaciones para la Nómina Educativa y el Gasto Operativo... Las reformas a la Ley de Coordinación Fiscal que establecieron el Fondo de Aportaciones para la Nómina Educativa y Gasto Operativo tuvieron como uno de sus fines el transferir la administración de la nómina de los servicios educativos que prestan las Entidades Federativas, de estas últimas a la Federación.

Véase nota informativa notacefp / 067 / 2014 del Centro de Estudios de la Finanzas Públicas LXII[en línea]Disponible en:
} 
gobernadores estatales para que cumplan con su responsabilidad y ordenen sus sistemas administrativos, pongan fin al pago a los aviadores y comisionados no educativos y dejen de pagar labores administrativas con dinero de la educación. A la Auditoría Superior de la Federación ASF para que finque responsabilidades, levante las demandas necesarias y recupere el dinero desviado ${ }^{15}$.

Si bien es cierto que en 2015 se otorgaba un plazo de dos años para la creación del andamiaje jurídico que diera formalidad al Sistema Nacional Anticorrupción SNA y un año más para que los estados hicieran lo propio -los estados debían homologar las leyes locales con las nacionales- de conformidad con los principios de su operación, a la fecha todavía existen estados que no se integran totalmente al sistema ${ }^{16}$.

De acuerdo con el Comité de Participación Ciudadana, que encabeza el $\mathrm{SNA}^{17}$, dentro de los requisitos que deben cumplirse a nivel nacional y estatal en la materia, algunas entidades y el mismo CPC que encabeza el sistema, no han cumplido del todo con sus responsabilidades establecidas por la ley.

Tres estados, por ejemplo, aún no han nombrado fiscal anticorrupción; cuatro no han concluido su adecuación legislativa; tres no cuentan con un Comité de

http://www.cefp.gob.mx/publicaciones/nota/2014/noviembre/notacefp0672014.pdf;( (consultado el 27 de febrero de 2019)

${ }^{15}$ Véase: Enfoque Noticias, El malgasto persiste en educación y genera exclusión e inequidad en el derecho a aprender: Mexicanos Primero,2016, pp,1[en línea]disponible en: https://enfoquenoticias.com.mx/noticias/el-malgasto-persisteen-educaci-n-y-genera-exclusi-n-e-inequidad-en-el-derecho-aprender; (consultado el 27 de febrero de 2019)

${ }^{16}$ La fecha límite venció el 18 de julio del 2018.

17 El Comité de Participación Ciudadana coordina los esfuerzos del sistema, apoyándose en el Instituto Nacional de Transparencia, Acceso a la Información y Protección de Datos Personales (INAI), la Secretaría de la Función Pública, la Auditoría Superior de la Federación (ASF), la Fiscalía Especializada de Combate a la Corrupción, el Tribunal Federal de Justicia Administrativa (TFJA) y el Consejo de la Judicatura Federal (CJF). 
Participación Ciudadana Estatal, y ocho estados aún no han nombrado un Secretario Técnico, y el mismo CPC no ha logrado homologar los lineamientos sobre las declaraciones patrimoniales de los funcionarios públicos.

Durante las reuniones nacionales de contralores, previas a la entrada en vigor del SNA, en el intercambio de experiencias se ponderaban algunas razones para que los sistemas estatales anticorrupción no pudieran homologar sus leyes en tiempo y forma: a. Falta de voluntad política del Ejecutivo estatal; b. División política y falta de acuerdos entre los partidos integrantes del Poder Legislativo local; c. Falta de recursos económicos para sustentar las entidades de nueva creación; y d. Falta de claridad respecto a la operación del sistema.

A dos años de esos intercambios, habría que revisar si existe la misma problemática; pero lo que sí está presente son los constantes anuncios de situaciones que involucran posibles hechos de corrupción, y una falta de claridad de cómo funcionará ahora el Sistema Nacional Anticorrupción, o si en la realidad lo hará.

\section{A MANERA DE CONCLUSIONES}

Es un acierto, sin duda, que un comité de ciudadanos sea quien encabece el SNA. Con ello se rompe una directriz de autoridad del Estado por sobre el tema del combate a la corrupción y la impunidad, para transitar a un modelo de corresponsabilidad sustentado en un marco legal. Algo nunca visto en México. Sin embargo no ha logrado mostrar su autoridad ante el Estado, y los avances logrados a la fecha solo han sido sectoriales ${ }^{18}$.

Ante la alternancia en la conducción del gobierno federal, el sistema entró en modo pasivo. Hoy, la mayoría de los mexicanos (51.2\%) cree que en México se

\footnotetext{
${ }^{18}$ El Sistema Nacional Anticorrupción da origen al Sistema Nacional de Fiscalización y al Sistema Nacional de Transparencia.
} 
puede acabar con la corrupción ${ }^{19}$ considerando la aceptación del actual presidente; y es difícil prever a ciencia cierta el futuro del sistema. Mientras tanto, si bien las exigencias sociales que dieron origen al SNA todavía persisten en México, el $52 \%$ de los mexicanos cree que la corrupción disminuirá en los próximos 12 meses $^{20}$.

La particularidad de esta situación, en relación con el caso hipótetico al principio de este ensayo mencionado, estriba, en tomar conciencia respecto a que mientras se revisan, entre otras, cuestiones como la transparencia en el manejo de los recursos para la Educación y se sigue combatiendo la corrupción,... Uriel, sigue esperando una oportunidad para hacer un plan de vida que lo saque de la pobreza.Corresponde entonces preguntar y responder ¿Dónde la encontrará... en la legalidad o en la ilegalidad?

\section{Referencias Bibliográfica}

ACEMOGLU Daron, ROBINSON James A., Porque Fracasan los Paises: Los Origenes del Poder, la Prosperidad y la Pobreza, Ed., Deusto,Barcelona España,2013, pp. 465

CÁMARA DE DIPUTADOS, LXII LEGISLATURA, Centro de Estudios de la Finanzas Públicas Nota informativa notacefp / 067 / 2014 [en línea]Disponible en:

http://www.cefp.gob.mx/publicaciones/nota/2014/noviembre/notacefp0672014.pdf;( (consultado el 27 de febrero de 2019)

COMITÉ DE PARTICIPACIÓN CIUDADANA, Política Nacional Anticorrupción,Propuesta de la Política Nacional Anticorrupción,Diciembre 2018,México,pp.23[en línea]disponible en: http://sumawebdesarrollo.com/cpcnacional_wp/wpcontent/uploads/2019/04/ Propuesta-de-la-Poli\%CC\%81tica-Nacional-Anticorrupcio\%CC\%81n.pdf

ENFOQUE NOTICIAS, El malgasto persiste en educación y genera exclusión e inequidad en el derecho a aprender: Mexicanos Primero,2016, pp.,1 [en línea]disponible en:

\footnotetext{
${ }^{19}$ Vease:Mexicanos contra la corrupción y la impunidad, Los Mexicanos Frente a la Corrupción y la Impunidad. Perspectivas y Prospectivas 2019, pp.9 [en línea]disponible en: https://contralacorrupcion.mx/mxfrentealacorrupcion/estudio/ 20 Ídem pp.8
} 
https://enfoquenoticias.com.mx/noticias/el-malgasto-persiste-en-educaci-ny-genera-exclusi-n-e-inequidad-en-el-derecho-aprender ; (consultado el 27 de febrero de 2019)

HERNÁNDEZ OCHOA,María Doris,La construcción del bien común, responsabilidad de todos,en Milenio Diario, S.A. de C.V.,2014 [en línea] disponible en: https://www.milenio.com/opinion/maria-doris-hernandezochoa/columna-maria-doris-hernandez-ochoa/la-construccion-del-biencomun-responsabilidad-de-todos; [consultado: 27 de febrero de 2019] INFORME A SONORA 2012; Sonora Educado, Gobierno del Estado de Sonora, 2012, pag. 4

MEXICANOS CONTRA LA CORRUPCIÓN Y LA IMPUNIDAD, Los Mexicanos Frente a la Corrupción y la Impunidad. Perspectivas y Prospectivas 2019, pp.9 [en línea]disponible en: https://contralacorrupcion.mx/mxfrentealacorrupcion/estudio/;(consultado el 14 de febrero de 2019)

MEXICANOS PRIMERO: (Mal)Gasto. Estado de la Educación en México 2013, Ed. Mexicanos Primero Visión 2030, México,2013, pp.4

PISARELLO, Gerardo. Los derechos sociales en el constitucionalismo democrático. Boletín Mexicano de Derecho Comparado, [S.I.], jan. 1998 [en línea]disponible en:

https://revistas.juridicas.unam.mx/index.php/derechocomparado/article/view/3546/4 242; (consultado el 27 febrero de 2019)

PRESIDENCIA DE LA REPÚBLICA, Plan Nacional de Desarrollo 2007-2012 [en línea]disponible en: http://pnd.calderon.presidencia.gob.mx/

PRESIDENCIA DE LA REPÚBLICA, Plan Nacional de Desarrollo 2013-2018 [en línea]disponible en: http://pnd.gob.mx/

SCHMELKES, S. Hacia una mejor calidad en nuestras escuelas. Biblioteca para la Actualización del Maestro. México, D.F. OEA/SEP,1992,pp97

TRANSPARENCY INTERNATIONAL, El índice de percepción de la corrupción muestra un estancamiento de la lucha contra la corrupción en la mayoría de los países [en linea]disponible en:

https://www.transparency.org/news/pressrelease/el_indice_de_percepcion_ de_la_corrupcion_2018; (consulltado el 27 de febrero de 2019)

Recibido 23 de Marzo 2019 